\title{
Genome-wide expression and network analyses of mutants in key brassinosteroid signaling genes
}

Razgar Seyed Rahmani ${ }^{1,2+}$, Tao Shi ${ }^{3,4+}$, Dongzhi Zhang ${ }^{4}$, Xiaoping Gou ${ }^{4}$, Jing Yi ${ }^{4}$, Giles Miclotte ${ }^{1,2}$, Kathleen Marchal ${ }^{1,2,5^{*}+}$ (D) and Jia Li $i^{4^{*+}}$

\begin{abstract}
Background: Brassinosteroid (BR) signaling regulates plant growth and development in concert with other signaling pathways. Although many genes have been identified that play a role in BR signaling, the biological and functional consequences of disrupting those key BR genes still require detailed investigation.

Results: Here we performed phenotypic and transcriptomic comparisons of A. thaliana lines carrying a loss-of-function mutation in BR/1 gene, bri1-5, that exhibits a dwarf phenotype and its three activation-tag suppressor lines that were able to partially revert the bri1-5 mutant phenotype to a WS2 phenotype, namely bri1-5/bri1-1D, bri -5/brs 1-1D, and bri1-5/bak1-1D. From the three investigated bri1-5 suppressors, bri1-5/bak1-1D was the most effective suppressor at the transcriptional level. All three bri1-5 suppressors showed altered expression of the genes in the abscisic acid (ABA signaling) pathway, indicating that ABA likely contributes to the partial recovery of the wild-type phenotype in these bri1-5 suppressors. Network analysis revealed crosstalk between BR and other phytohormone signaling pathways, suggesting that interference with one hormone signaling pathway affects other hormone signaling pathways. In addition, differential expression analysis suggested the existence of a strong negative feedback from BR signaling on BR biosynthesis and also predicted that BRS1, rather than being directly involved in signaling, might be responsible for providing an optimal environment for the interaction between BRI1 and its ligand.
\end{abstract}

Conclusions: Our study provides insights into the molecular mechanisms and functions of key brassinosteroid (BR) signaling genes, especially BRS1.

Keywords: Brassinosteroid signaling, Expression analysis, Systems biology, Network analysis, Arabidopsis

\footnotetext{
* Correspondence: kathleen.marchal@ugent.be; lijia@lzu.edu.cn

${ }^{\dagger}$ Razgar Seyed Rahmani and Tao Shi contributed equally to this work.

Jia Li and Kathleen Marchal contributed equually.

'Department of Plant Biotechnology and Bioinformatics, Ghent University,

Ghent, Belgium

${ }^{4}$ Ministry of Education Key Laboratory of Cell Activities and Stress

Adaptations, School of Life Sciences, Lanzhou University, Lanzhou 730000,

China

Full list of author information is available at the end of the article
}

(c) The Author(s). 2021 Open Access This article is licensed under a Creative Commons Attribution 4.0 International License, which permits use, sharing, adaptation, distribution and reproduction in any medium or format, as long as you give appropriate credit to the original author(s) and the source, provide a link to the Creative Commons licence, and indicate if changes were made. The images or other third party material in this article are included in the article's Creative Commons licence, unless indicated otherwise in a credit line to the material. If material is not included in the article's Creative Commons licence and your intended use is not permitted by statutory regulation or exceeds the permitted use, you will need to obtain permission directly from the copyright holder. To view a copy of this licence, visit http://creativecommons.org/licenses/by/4.0/ The Creative Commons Public Domain Dedication waiver (http://creativecommons.org/publicdomain/zero/1.0/) applies to the data made available in this article, unless otherwise stated in a credit line to the data. 


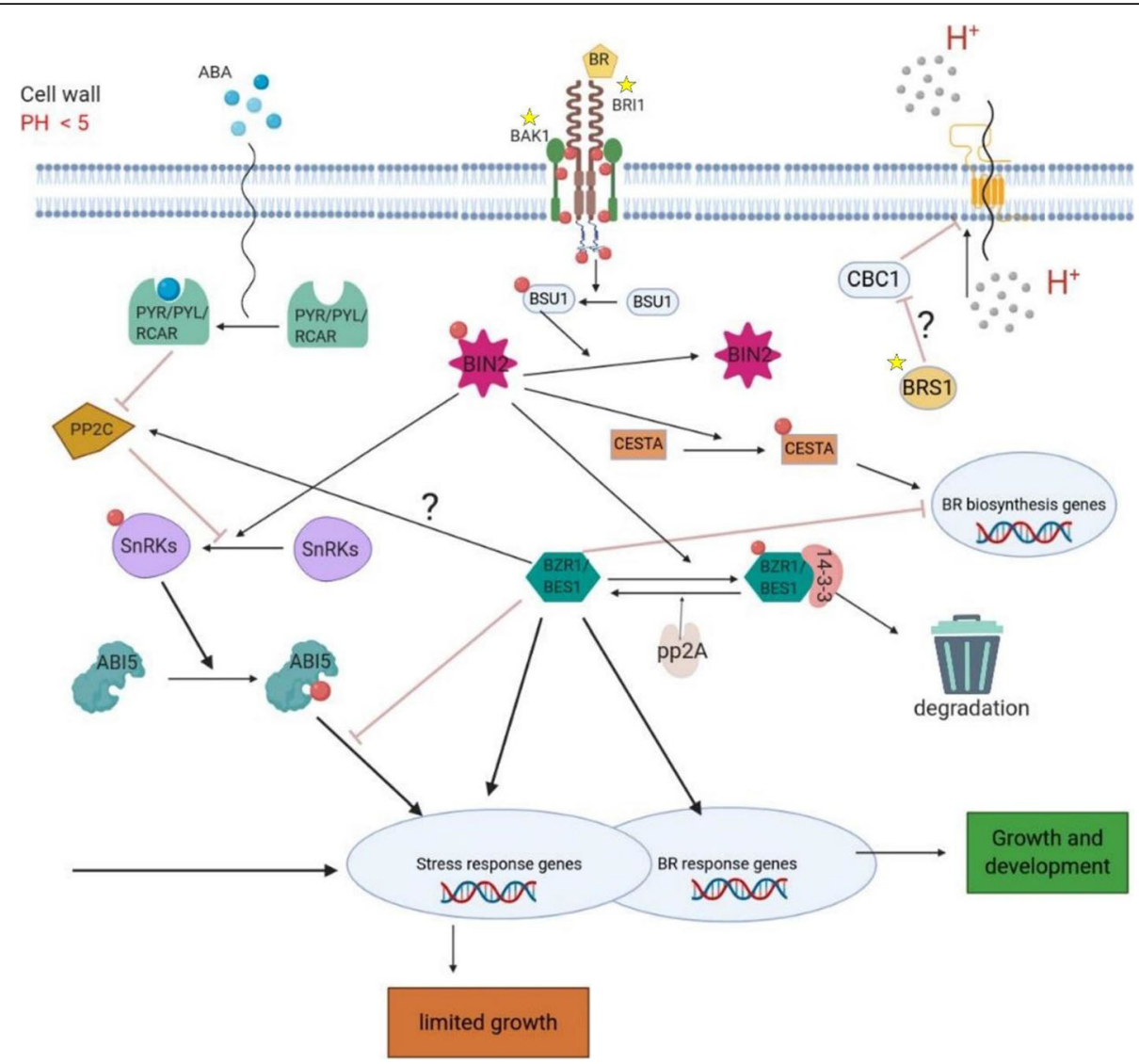

Fig. 1 Schematic overview of the BR signaling cascade and the results of this study. The figure provides a simplified scheme of BR signaling based on [4, 5]. The genes studied in this work are indicated by a yellow star. Binding of BRs to the BRI1/BAK1 receptor triggers the phosphorylation/dephosphorylation signaling cascade that leads to the deactivation (dephosphorylation) of BIN2. The effects of BIN2 and BZR1/BES1 on BR-biosynthesis genes are depicted. The overlap between stress-response and BR response genes and the dual effect of BZR1/BES1 on stress response genes is also shown. The question marks indicate missing links that have been suggested based on the result of the present study. The hypothetical inferred role for BRS1 in providing a better condition for BRI/BAK1/BR binding by generating a more acidic environment is shown on the top right-hand side. The compensatory pathway resulting in the over-expressing expression of PP2C mediated by ABA is shown on the left-hand side. (Created with BioRender.com)

\section{Background}

Brassinosteroids (BRs) are essential plant hormones, regulating multiple processes amongst which plant growth, flowering, senescence, and seed germination [1]. BR biosynthetic and signaling mutants display aberrant morphological phenotypes such as dwarfism, reduced fertility, impaired photomorphogenesis, and altered vascular development $[2,3]$. Whereas the phenotypes of mutants in BR biosynthetic genes can be rescued by the application of exogenous BRs, this is not the case for strains carrying mutations in genes responsible for BR signal perception and transduction. Hence these latter strains are referred to as BR insensitive (bri) mutants $[1$, 3]. In the BR signaling pathway (Fig. 1), BRs are perceived by membrane-localized leucine-rich-repeat-receptor kinase BRI1 or by the BRI1-like homologs, BRL1 and BRL3 [6, 7]. After binding to BRs, BRI1 and its coreceptor BRI1-Associated Receptor Kinase 1 (BAK1) phosphorylate each other. This results in triggering a cytoplasmic phosphorylation/dephosphorylation signaling cascade which deactivates the GSK3-like kinase BRASSINOSTEROID INSENSITIVE 2 (BIN2) through dephosphorylating $[4,5]$. Upon BIN2 deactivation, the downstream transcription factors, BRASSINAZOLERESISTANT1 (BZR1) and BR-INSENSITIVE-EMS-SUPPRESSOR1 (BES1) are dephosphorylated by PP2A (PHOSPHATASE 2A). This results in their disassociation from 14-3-3 proteins, causing them to get activated and regulating a range of downstream genes involved in various aspects of plant growth and development [8-10]. In the absence of BRs, BIN2 is active (phosphorylated) and it prevents the activation of BZR1 and BES1. Because BRI1 is the core receptor of BRs, mutants of BRI1 have been used as genetic background to identify suppressors, i.e. other genes that when mutated, suppress the bri1 phenotype and thus may play a role in BR signaling. For example, the function of BZR1 has been unveiled by using the null allele of BRI, bri1-116 
[11]. The weak mutant of bri1, bri1-5, can be rescued by overexpression of $B A K 1$ and BRI Suppressor 1 (BRS1) $[3,12]$. BRS1 is a secreted member of the serine carboxypeptidase (SCP) family [3]. The fact that overexpression of BRS1 can suppress two weak BRI1 extracellular domain mutants, bri1-5 and bri1-9, but not the strong cytoplasmic domain mutant bri1-1, implies that $B R S 1$, unlike the downstream genes, $B Z R 1$ or $B E S 1$, may function upstream of the BR signaling pathway or in a close regulatory relationship with BRI1 [3]. Moreover, three of the five overexpressed BRS1's homologs amongst which ECS1 (Extra Carpels and Seeds 1) can also partially suppress the phenotype of the bri1-5 mutant observed in leaves. Overexpression of BRS1's homologs also increases the number of carpels and seeds, confirming the role of BRS1 and its homologs in the BR signaling [13]. Yet, the detailed mechanism of how BRS1 potentially interacts with other BR genes in order to maintain balance in BR signaling is still unknown.

Some genes involved in BR signaling are also involved in other processes, such as stress response, and can act independently of the presence of BRs. Several studies found that bes1-1D and bzr1-1D backgrounds are not responsive to exogenous BRs, suggesting that BES1 and $B Z R I$ have also other functions than BR signaling [14, 15]. In another study, BAK1 was found to work together with Flagellin-Sensitive 2 (FLS2) during pathogen defense programmed cell death independently of BR signaling $[14,16-18]$. In addition, SERK1 and SERK2, the homologs of BAK1 play a role in male microsporogenesis, also independently of BR signaling [19]. Some bri1 mutants show in addition to reduced growth, an increased stress-tolerance, further confirming the complexity and dosage sensitivity of BR signaling and regulation [20, 21]. Transcriptomic studies and network analysis have shown to be effective in uncovering the expression and biological consequences of gene mutants, and have successfully been applied to study several BR genes such as BRI1 and BES1 [22]. Therefore, in the present study, we applied a similar strategy to elucidate the role of $B R I 1, B A K 1$, and $B R S 1$ in regulating/restoring the response to BRs and/or in other functions independent of BR signaling.

\section{Results}

bri1-5/bak1-1D, bri1-5/brs 1-1D and bri1-5/bri1-1D partially reconstitute bri1-5 gene expression

To better understand the molecular mechanisms of key BR signaling genes, we performed a phenotypic screening and expression analysis of bri1-5 and its three activation-tag suppressors along with their corresponding wild-type, WS2. Two suppressor strains bri1-5/ bak1-1D and bri1-5/brs-1D were obtained from [12]. An additional bri1-5/bri1-1D mutant was generated in the framework of the current study (see Methods). Sequencing the BRI1 flanking region from the suppressor bri1-5/bri1-1D showed that the activation tag was inserted $534 \mathrm{bp}$ downstream of the BRI1 gene (Supplementary Fig. 1-A). All suppressor mutants were shown to indeed overexpress the activation tagged gene as confirmed by Real-Time qPCR (RT-qPCR) (Table S1). Phenotypically, all bri1-5 suppressors (bri1-5/bak1-1D, bri1-5/brs1-1D, and bri1-5/bri1-1D) lines displayed larger seedlings than the bri1-5 mutant, but still significantly smaller than the WS2 (Fig. 2). Of all suppressor mutants, the bri1-5/bak1-1D line best approximated the growth phenotype of the WS2, and its larger seedling seemed to be mainly the effect of its larger root length and to a lower extent of its larger hypocotyl length (both of which were significantly larger than the bri1-5 mutant). The contribution of the epidermal cell length in recovering the bri1-5 is marginal in the bri1-5/bak1$1 D$ (line with the largest seeding) but seems much more pronounced in the bri1-5/brs1-1D (Fig. 2, Supplementary Fig. 1: B-F). This indicates that in the bri1-5/brs1$1 D$ mechanisms other than those in the bri1-5/bak1-1D line play a role in alleviating the bri1-5 phenotype.

To gain insight into which pathways in each of the studied lines were responsible for recovering the bri1-5 growth phenotype to wild-type level, we performed gene expression analysis. All suppressor lines, together with the wild-type (WS2) and the bri1-5 background were sampled at a 7-day seedling stage. To assess the reproducibility of the expression analysis, we measured the extent to which the expression profiles of replicate samples were similar using Principal Component Analysis (PCA): PCA indeed showed that the largest fraction of the variation in gene expression between the samples could be assigned to differences in genetic background and not to differences between replicates of the same genetic background, confirming the reproducibility (Fig. S2). In addition, microarray results were confirmed using RT-qPCR for a randomly selected set of differentially expressed genes (Fig. S3).

We determined for each mutant line its differential expression versus the same common reference i.e. the expression state in WS2, resulting in a total of 1413 differentially expressed genes (Additional file 1). The Venn diagram represented in Fig. 3 shows to what extent the different lines share the same differentially expressed genes (aberrantly expressed versus the WS2 control). Fig. 3 and the scatter-plots in Fig. S4 (A-C) show that of all suppressor lines, bri1-5/bak1-1D could restore the largest number of genes that were affected in expression in bri1-5 (about two-thirds of the genes that were differentially expressed in bri1-5 were no longer differentially expressed in bri1-5/bak1-1D). This is in line with its observed phenotypic behavior as indeed 

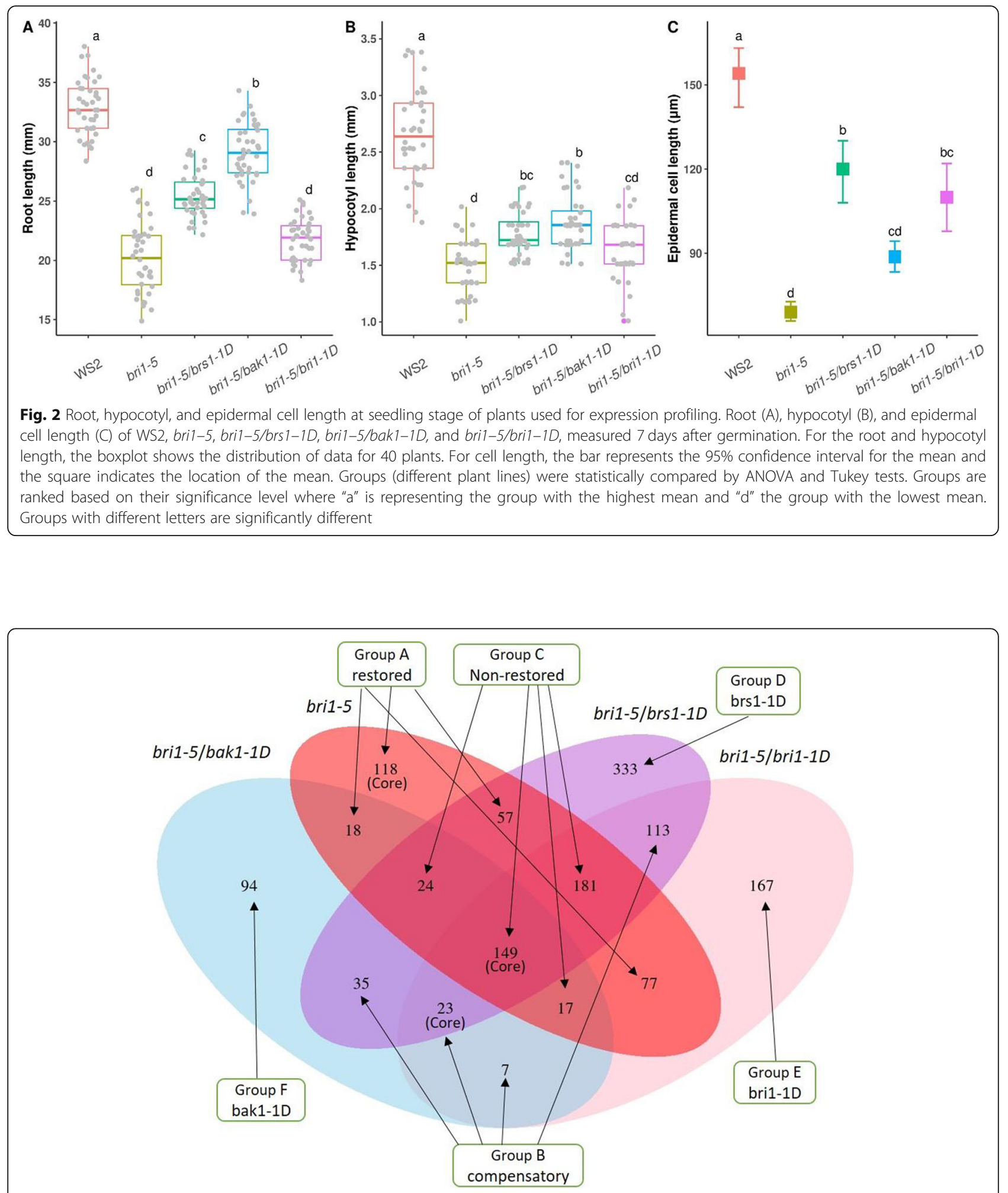

Fig. 3 Differentially expressed genes (DEGs relative to WS2) being compared between bri1-5 and its three suppressors. Group A (restored genes, 270 genes): genes differentially expressed in the bri1-5 mutant but no longer in at least two of the suppressor lines; Group B (compensatory genes, 178 genes): genes that are differentially expressed in at least two suppressors but not in bri1-5; Group C (genes that were not restored, 371 genes): Genes that are aberrantly expressed in bri1-5 and at least two of the suppressor lines. Group D (333 genes), E (167), and F (94 genes) contain genes that are exclusively differentially expressed in respectively the bri1-5/brs 1-1D, bri1-5/bri1-1D, and bri1-5/bak1-1D suppressor lines. The "core" below the number indicates the most reliable set for the group. The total number of potentially interesting genes is 1430 
bri1-5/bak1-1D seems to also phenotypically best compensate for the bri1-5 mutation.

The Venn diagram in Fig. 3 also shows that the bri15/brs $1-1 D$ and bri1-5/bri1-1D lines share the largest fraction of similarly affected genes. The latter is also illustrated in Fig. S4 panel D-F which shows that from all pairwise comparisons between suppressor lines, the level of differential expression relative to the mutant bri1-5 is most correlated between the suppressor lines bri1-5/ brs $1-1 D$ and bri1-5/bri1-1D (i.e. $R^{2}=0.20$ ). This suggests a similar role for BRI1 and BRS1 in the BR signaling pathway. Note that in Fig. S4 D-F, rather than performing a direct correlation analysis of the expression between two mutant lines, we performed correlation analysis with the expression of each mutant line relative to the same reference (expression in bri1-5). In this way, the correlation analysis is driven by the expression of the genes that change their expression relative to bri1-5. Although this results in lower correlation values than when directly comparing the expression values of the mutant lines, it better reflects the consistency between mutant lines in restoring genes affected in the bri1-5 mutant.

To confirm the extent to which the different suppressor strains molecularly restore the defects in the bri1-5 mutation, we compiled a list of marker genes representative of downstream pathways affected by BR signaling (Additional file 2). This consisted of 233 marker genes that were according to literature regulated by BR signaling (genes that became up or down-regulated upon treatment with exogenous BRs or by overexpressing the BR signaling genes). Of those marker genes, only those that were significantly affected in the bri1-5 line were retained in order to identify the mutant line that best suppresses the bri1-5 mutation (96 marker genes). Fig. 4 and Fig. S5 show how the expression of these genes is, as compared to WS2 affected in the bri1-5 mutant and how some of those genes got restored in the suppressor mutants. These results confirm what we observed based on the global expression analysis, i.e. that the bri1-5/ bak1-1D restored the bri1-5 affected marker genes to the largest extent, and that molecularly the bri1-5/brs1$1 D$ and bri1-5/bri1-1D mutant tend to behave more similarly in restoring the same marker genes.

\section{Identifying compensatory and restoring pathways}

Pathway analysis (see Methods) unveiled the pathways overrepresented amongst the differentially expressed gene sets in each of the mutant lines. Fig. S6, S7 and S8 and Table $\mathrm{S} 2$ show a number of pathways that are differentially expressed in both bri1-5 and all of the suppressor lines. These represent the pathways that are responsible for the aberrant growth phenotype in the bri1-5 mutant and that could not entirely be restored or compensated for in the suppressor lines. Among others, pathways related to cell wall synthesis (cell wall cellulose synthesis), protein and lipid metabolism can explain the residual discrepancy between the WS2 growth phenotype and the suppressors.

We assumed that if the suppressor strains alleviate the phenotype of the bri1-5 mutant, they could do so because they either restore the pathways disrupted in the bri1-5 mutant to wild-type levels or they induce genes that compensate for the bri1-5 affected pathways. Both mechanisms are reflected in the expression data. Processes that are aberrantly expressed in the bri1-5 mutant, but not in any of the suppressor lines, represent pathways that are restored to WS2 levels in all of the suppressors. This seems to be the case for some genes related to cytochrome P450 oxidase (Table S2). The fact that they are restored (or not significantly affected) in any of the suppressors indicates they might be essential for the recovery of the WS2 phenotype. Interestingly, the genes related to "glutathione S transferases" (Fig. S7, Table S2) are largely down-regulated in the bri1-5 mutant, restored to normal in bri1-5/bri1-1D and bri1-5/ bak1-1D and up-regulated as compared to WS2 levels in the bri1-5/brs $1-1 D$ suppressor, indicating that some overcompensation is needed for this pathway in the bri1-5/brs1-1D background in order to restore the bri1-5 phenotype. In addition, ABA-related metabolism (Fig. S8 and Table S2) seems to have been affected by all suppressors, but at least not to a significant level in the bri1-5 mutant. Therefore, ABA signaling seems to represent a compensatory pathway, i.e. a pathway that needs to be triggered in the suppressor strains in order to restore the bri1-5 affected pathways and phenotype.

As less than 5000 genes can be mapped using pathway analysis, we performed a more elaborate analysis using a network-based approach. Network analysis provides an intuitive way of combining expression data with prior information on known molecular interactions or already available functional data [23, 24]. This approach first maps candidate genes, that are identified through expression analysis, on an integrated molecular interaction network. Then, it identifies subnetworks that connect as many candidate genes as possible [24]. By leveraging candidate genes identified through expression analysis with known interaction information, spuriously identified candidate genes can be removed as they will not be part of the subnetworks. In addition, genes relevant to the process of interest that are themselves not regulated at the level of expression are indirectly identified by being part of a connected component/subnetwork to which also many of the candidate genes belong. Such an integrated analysis provides a more comprehensive view of the process of interest. Here, we applied such an integrated network-based strategy to gain a more in-depth insight into the molecular mechanisms through which 


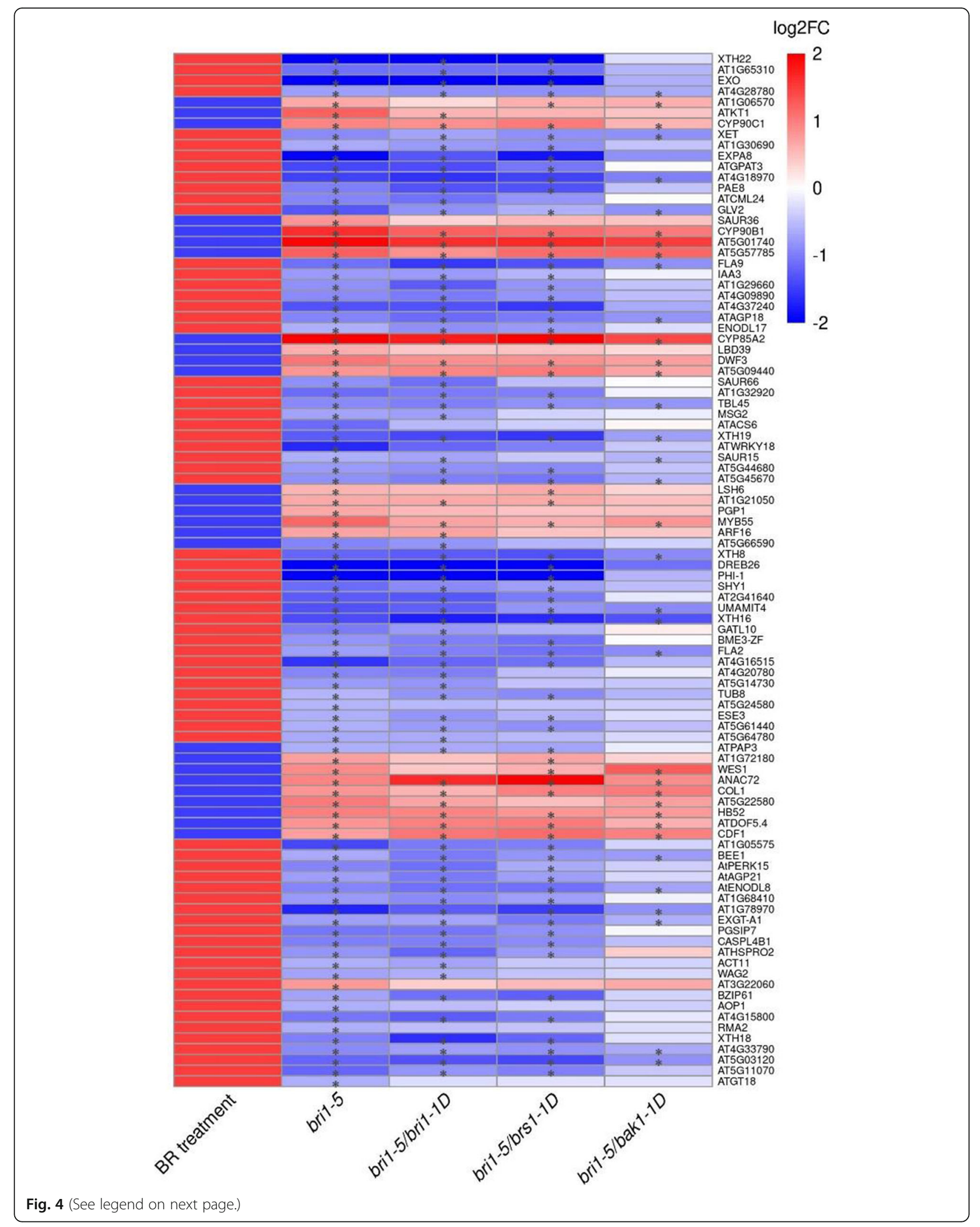


(See figure on previous page.)

Fig. 4 Expression behavior of marker genes representative of downstream BR signaling pathways. Column BR treatment: colors indicate whether a gene was reported to be up (red) or down (blue) regulated according to literature upon treatment with exogenous BRs or in a line containing a gain-of-function mutation in a BR signaling gene. Genes were only selected as representative for downstream BR signaling if the up/down regulation of their expression was confirmed by at least 5 independent references and also affected in the bri1-5 line of our study (compared to WS2). Columns bri1-5, bri1-5/bri1-1D, bri1-5/brs 1-1D, bri1-5/bak1-1 indicate whether the genes were found to be up or down-regulated compared to WS2 according to our expression data. Color scale indicates whether a gene is up-regulated (red), down-regulated (blue), or not differentially expressed (white). $A$ * indicates that the adjusted $p$-value $<0.05$

the suppressor lines can restore the bri1-5 phenotype to WS2 levels. To perform this network analysis, we started from the gene sets depicted in Fig. 3.

\section{Involvement of hormone signaling in alleviating the bri1- 5 phenotype}

To study the interaction between non-restored, restored, and compensatory pathways in more depth, we combined the following gene sets for network analysis (see Fig. 3): i) genes that were most likely restored in the suppressors (genes of group A i.e. the genes with altered expression in the bri1-5 mutant, but restored to WS2 level in at least 2 suppressors), ii) genes that were compensatory in most of the suppressors (genes of group B i.e. the genes not differentially expressed in the bri1-5 mutant, but differentially expressed in at least two suppressor strains) and iii) genes altered in the bri1-5 mutant that most likely were not restored in the suppressors (genes of group $C$ i.e. the genes, differentially expressed in the bri1-5 mutant and at least two of the suppressor strains). This combined set of genes (789 genes) is referred to as the set of seed genes or the genes we want to maximally connect on the interaction network.

Network analysis (see Material and Methods) identified 8 sub-networks (Fig. 5) containing the set of seed genes that could be connected through the interaction network. These subnetworks contain not only seed genes, but also connector genes. These are genes that are not differentially expressed themselves, but that are still recovered by the network analysis, because of their high connectivity with seed genes. As they are needed to connect seed genes in the network, they are most likely involved in the same processes as the seed genes. The subnetworks were annotated based on their enrichment in known GO functions (being enriched in respectively negative regulation of $\mathrm{ABA}$, response to auxin, fatty acid metabolism process, developmental process, oligopeptide transport, response to ROS, BR homeostasis, and Ethylene activated signaling (Fig. 5)). This indicates that these are the pathways that contribute to alleviating bri1-5 signaling deficiency in the suppressor strains.

In-depth analysis shows that the subnetwork enriched in ABA signaling (Fig. 5, subnetwork 1) contains several known negative regulators of ABA signaling: HAI1, $H A B 1 A B I 1, A B I 2$, and PP2CA acted as compensatory genes: these were are up-regulated in at least two bri1-5 suppressor lines compared to wild-type, but were not affected in the bri1-5 mutant (HAI1 and HAB1 being significantly up-regulated in all suppressor mutants; $A B I 1$, $A B I 2$, $P P 2 C A$, being significantly up-regulated in two suppressors, see Fig. S9); In addition, HAI2 was affected in the bri1-5 line, but could not be restored in at least two suppressors (non-restored gene), and HAB2 was identified as a connector node.

Interestingly, several targets of the $\mathrm{ABA}$ signaling pathway (DTX50, HVA22D, PUB19, COR15B, next to $H A I 1, H A B 1)$ were identified as differentially expressed in all three suppressors (identified based on a GO enrichment of the core of group $B$, but not in bri1-5). This indicates that ABA signaling has indeed been affected in the suppressor strains to compensate for the bri1-5 signaling deficiency. Of these, DTX50, HVA22D, PUB19, COR15B could not be connected by PheNetic on the interaction network, implying they are either not annotated in the interaction network $(C O R 15 B)$ or quite distantly located from each other in the network.

The aforementioned negative regulators of ABA signaling in subnetwork 1 belong to the protein phosphatase $2 \mathrm{C}$ (PP2C) gene family which has nine members in total (HAI2, HAB2, HAB1, HAI3, PP2CA, ABI1, AHG1, ABI2, AHI1). $\mathrm{PP} 2 \mathrm{C}$ is known to indirectly repress ABI5, the main activator of ABA signaling [25]. PP2C is also known to repress BIN2 activity [4, 5]: as BIN2 activates ABI5 by phosphorylating SnRKs $[4,5]$, repressing ABA signaling by PP2C via blocking SnRKs phosphorylation seems to compensate for the deficiency in BRI1 mediated signaling (Fig. 1). The subnetwork enriched in ABA signaling (network 1) also contains members of the PYR/PYL/RCAR family as connector genes (RCAR5, RCAR6, RCAR7, RCAR10, RCAR14, PYL8). The PYR/PYL/RCAR family constitutes the receptor of ABA signaling and promotes the activation of SnRKs by repressing PP2C $[15,26]$. The fact that the SnRKs (SnRK2.5) and PYR/ PYL/RCAR genes were identified as connector genes implies that they are likely involved in the pathways that connect the affected, restored, and compensatory genes of subnetwork 1 . They are most likely not primarily regulated at the expression level, given their role in phosphorylation-mediated signaling $[5,27]$. This explains why they were detected as connector genes and not retrieved by differential expression analysis. 


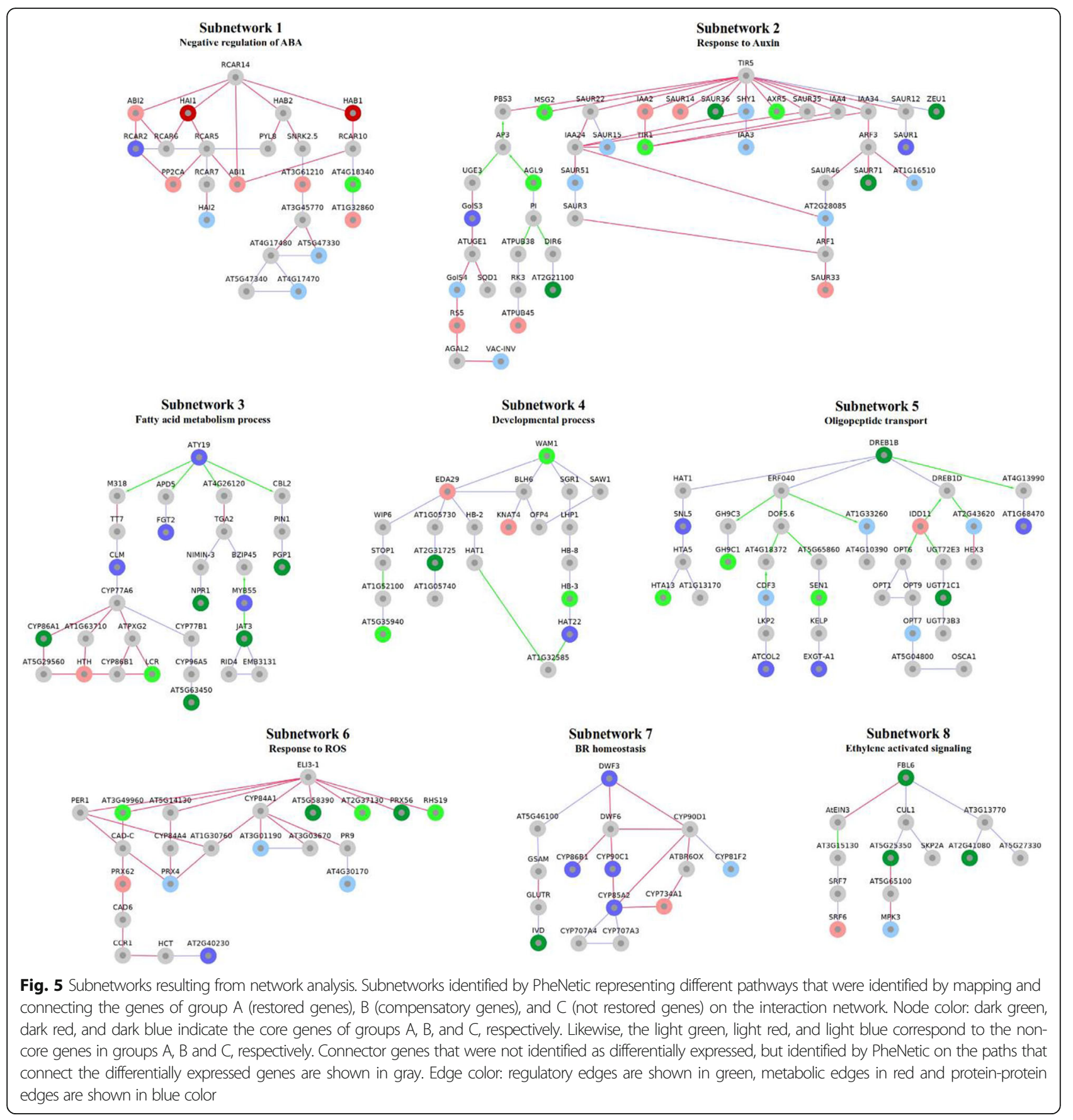

We could not find any link in the literature to explain how $P P 2 C$ can be up-regulated by BR signaling in order to repress $A B A$ signaling. It seems that there exist some missing links between BZR1/BES1 (or downstream TFs) and the $P P 2 C$ gene family. By partially recovering BR signaling in the suppressor lines, one would expect that $P P 2 C$ gene expression levels restore to WS2 expression level. However, they appear to become up-regulated in suppressors, indicating that further compensatory repression of the ABA signaling is required in order to restore the bri1-5 phenotype.
Other than the ABA subnetwork, subnetworks related to other hormone signaling processes like auxin signaling (subnetwork 2), ROS signaling (subnetwork 6), and ethylene signaling (subnetwork 8) were also detected. It is well known that crosstalk between these phytohormone signaling pathways exists $[28,29]$. Hence, interfering with one pathway e.g. $A B A$ signaling through $B R$ signaling might affect other phytohormone pathways as well. Based on the pathway analysis using MapMan and the network analysis we can conclude that ABA 
signaling is mostly a compensatory pathway (genes indicated in red color in Fig. 5 represent compensatory genes), whereas auxin, ethylene, and ROS signaling pathways are at least partially restored to the WS2 level (genes indicated in green color in Fig. 5 represent restored genes). However, restoring those pathways to the WS2 level seems to also depend on the presence of at least some compensatory genes (genes indicated in red color in Fig. 5 represent compensatory genes). The remaining subnetworks are enriched for fatty acid metabolism (subnetwork 3), developmental processes (subnetwork 4), and oligopeptide transport (subnetwork 5) confirming that all suppressors could partially restore some affected primary metabolic pathways. This is in line with the MapMan results and the recovered phenotypes.

\section{Negative feedback of BR signaling on BR biosynthesis}

The network result shows that the BR biosynthesis (subnetwork 7) is not well recovered in any of the suppressors. This subnetwork contains 4 genes (CYP90C1, CYP90B1/ DWF4, CYP85A2, CYP90A1/DWF3) that are differentially expressed in the bri1-5 lines and all of the suppressors. Those genes, belonging to the cytochrome P450 superfamily play a role in BR biosynthesis by converting the sterol "campesterol" to BRs [30]. CYP708A3, another BR biosynthesis gene was found to be differentially expressed in all suppressors and the bri1-5 mutant (Fig. 6). However, as this gene was not present in the interaction network, it was missed by the network analysis. Unlike the four aforementioned cytochrome P450 genes, the molecular function of the CYP708A3 gene is still unknown. Interestingly it shows an expression pattern that is anticorrelated to that of the other cytochrome P450 genes (Fig. 6). This result along with the fact that CYP708A3 is known to be up-regulated by exogenous brassinolide (BL) treatment $[31,32]$ suggests it acts as an inhibitor of BRs biosynthesis.

The fact that the expression of BR biosynthetic genes is affected by mutations in BR signaling genes points towards the existence of negative feedback of BR signaling on BR biosynthesis. If indeed negative feedback exists between BR signaling and biosynthesis, this feedback should be reflected in quantitative differences in

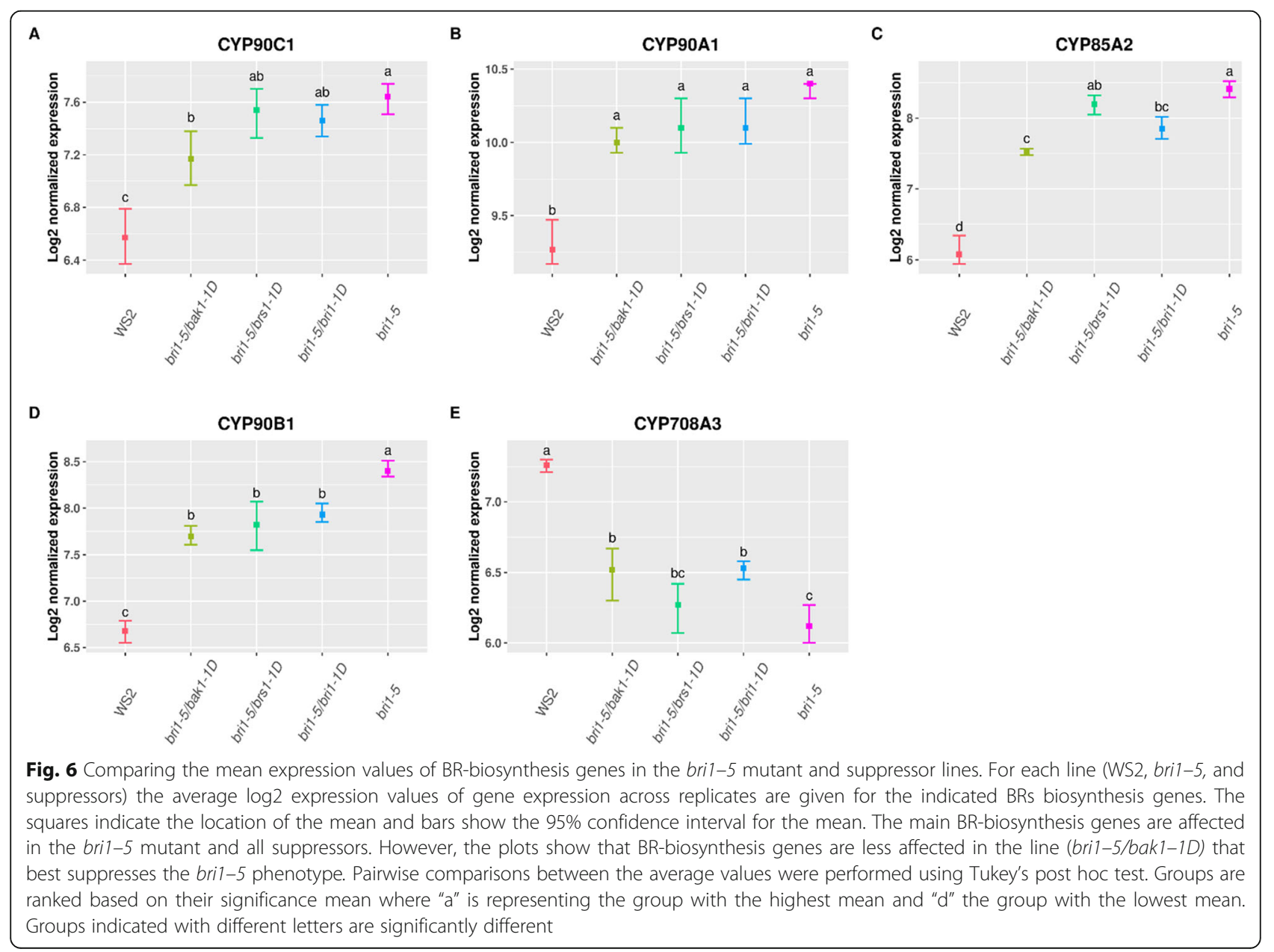


overexpression of the BR signaling and biosynthesis genes in the bri1-5 and suppressor mutants. The better the signaling can be restored in the suppressors (as reflected by the phenotype), the less we expect the expression of the BR biosynthesis to be aberrant. We indeed found that the expression of the BR-biosynthesis genes (CYP90C1, CYP90A1, CYP85A2, CYP90B1, $C Y P 708 A 3)$ are less affected in the strains that better mimic the wild-type phenotype (see Fig. 6, the best suppressor of bri1-5, bri1-5/bak1-D, shows the lowest expression change of the biosynthesis genes). This further supports the existence of negative feedback from $B R$ regulation on $B R$ biosynthesis: a more sustained BR signaling results in decreased BR biosynthesis, whereas suboptimal BR signaling is compensated for by higher transcriptional activity of BR biosynthetic genes.

\section{Link between stress response and BR signaling}

Next, we analyzed the genes that were uniquely altered in each of the suppressors, i.e. the suppressorspecific compensatory genes (genes of group $\mathrm{D}$, group E, and group F, respectively). Based on GO analysis we mainly found stress related processes to be overrepresented in each of the groups. Genes involved in these stress related processes seem to be scattered over the interaction network as they could not be recovered as well delineated subnetworks, indicating that different stress-related genes are induced in the different lines. According to the literature, there is crosstalk between BR signaling and signaling by other hormones in response to stress, especially via $A B A$ and auxin signaling [28]. In the absence of BRs (or low amounts of BRs), BIN2, affects ABA and auxin signaling, resulting in the induction of stress response genes $[4,5,15]$. On the other hand, some stressresponse genes are known to be targets of $B Z R 1$ and BES1 [5, 15] (Fig. 1), indicating that also when BRs levels are high, stress response genes can be activated. These observations show that balanced BRs levels are needed for normal growth and that deviation from the optimal levels (either too high or too low) would activate stress response mechanisms. We observed that by partially recovering bri1-5 signaling deficiency by suppressors mutants, the transcript level of some stress-response genes is restored to normal, but other stress-response genes become induced (Additional file 3: GO enrichment for genes exclusively differentially expressed in each suppressor, "GO_only bri1-5", "GO_only_bri1-1D”, “GO_only_bak1-1D”, "GO_only_brs1-1D"). This observation is in line with this complex effect of BRs and BR signaling on stress response pathways.
Iron ion homeostasis, ferroxidase, and glutathione transferase activity are identified as compensatory mechanisms unique to the bri1-5/brs 1-1D suppressor Unlike for BRI1 and BAK1, much less is known about the role of BRS1 in BR signaling. Therefore we had a closer look at genes of group D which are exclusively differentially expressed in bri1-5/brs $1-1 D$ mutant and hence comprise compensatory pathways specific for bri1-5/brs1-1D. GO enrichment showed that the genes of this group (group D, 333 genes) are not only overrepresented in stress related processes (see above) but also in glutathione transferase (up-regulated), (Fig. 7). This overrepresentation in glutathione transferase is in line with the MapMan results. These results showed how in the bri1-5/brs $1-1 D$ mutant the expression of the glutathione transferase was not only restored as compared to the other suppressor lines, but even overcompensated as compared to WS2 levels (Fig. S7). We also found that several members of the CCAAT-binding factor complex (CBC) (NFYA2, NFYA3, NFYA6, NFYA10) were uniquely up-regulated in bri1-1D/brs1-1D (Fig. 7 and Fig. S9). Members of this complex have been associated with the control of iron homeostasis in Candida glabrata [33]. In addition, iron ion homeostasis/ferroxidase activity was also found to be down-regulated, specifically in the bri1-5/brs1-1D. In the ferroxidase reaction, four $\mathrm{H}^{+}$ are used to catalyzes the oxidization of $\mathrm{Fe}^{2+}$ to $\mathrm{Fe}^{3+}$, repressing this reaction results in the accumulation of $\mathrm{H}^{+}$which can be transported to the apoplast via plasma-membrane pumping mediated by ATPase ( $\mathrm{H}+$-ATPase transporters) [34]. Accordingly, we also found that the main inhibitor of $\mathrm{H}^{+}$-ATPase transporters, $C B C 1$, was significantly down-regulated in bri1-5/brs1-1D (fold change - 1.7, adj $p$-value $8.36 \mathrm{e}$ 06), but not in the other suppressors. This implies that $\mathrm{H}^{+}$-ATPase transporters are more active in bri15/brs1-1D to export $\mathrm{H}^{+}$from cytosol into apoplast, making the apoplast more acidic (Fig. 1). In line with this hypothesis, the up-regulated glutathione transferase activity in the brs1-1D mutant (Fig. S9) might be essential to compensate for the more acidic environment in the bri1-5/brs $1-1 D$ and would be required for maintaining redox homeostasis. In addition, we hypothesize that the observed acidification could generate a cellular environment that improves BRI1-BRs binding or BRI1-BAK1 dimerization and hence contributes to restore the bri1-5 mutant phenotype.

\section{Discussion}

In this study, we explored the alteration of gene expression in BR signaling mutants to better understand BR signaling and the functions of key BR genes. 


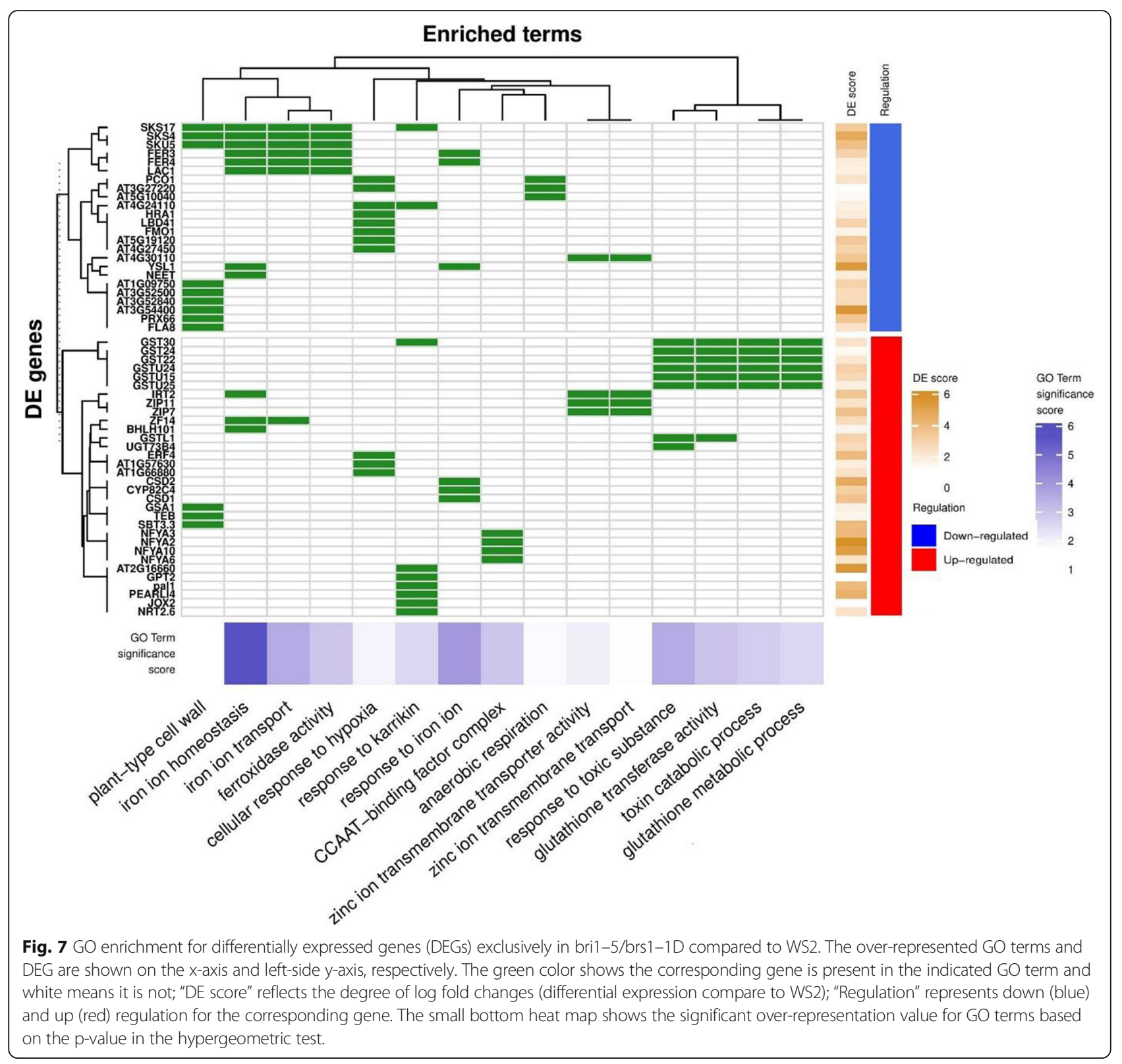

\section{Crosstalk between BR signaling, other hormone signaling} pathways, and primary metabolites

Our analysis identified ABA signaling as a mainly compensatory pathway, and hormone signaling pathways related to ethylene, auxin, and ROS, as pathways involved in partially restoring the bri1-5 expression phenotype to WS2 levels. It is also obvious that restoring pathways can depend on the presence of compensatory genes in the same pathway. The crosstalk between BR signaling and signaling by ABA observed in our study is in line with the literature [35]. The serine-threonine kinases SnRK2.2 is the main positive regulator of ABA signaling by regulating key TFs such as $S L A C 1, K A T 1$ [36, 37]. PHOSPHATASE 2C (PP2C) represses the SnRK2.2 by blocking its phosphorylation mediated by BIN2 (Fig. 1). In the presence of ABA, the complex of PYR/PYL/RCAR inactivates the PP2C by blocking its substrate's entry [38]. The activated SnRK2 phosphorylate ABI5 leading to the activation of downstream ABA-dependent mechanisms. In the absence of BRs (or BR signaling deficiency like bri1-5), SnRK2.3 can mimic the presence of ABA in triggering ABA signaling, once it is phosphorylated by BIN2 [25]. This means that repressing ABA signaling (repressing SnRK2) can compensate for the BR signaling deficiency. In line with our result, a recent study showed that overexpression of $A B I 1$ or $A B I 2$, which encodes the negative regulator of $\mathrm{ABA}$ signaling could promote $\mathrm{BR}$ signaling [35]. Network analysis suggested that all bri1-5 
suppressor strains suppressed the ABA signaling (SnRKs) through up-regulating its negative regulator, PP2C (Fig. 1).

Links between BR signaling and auxin we observed are also supported by literature and in line with the observed phenotype of the bri1-5 mutant. ARFs (auxin response factors) are transcription factors that affect root and shoot elongation [29]. In the presence of BRs, BZR1 and BES1 enhance the DNA-binding activity of the auxin response factors ARF6 and ARF7 to promote auxin response $[39,40]$. This explains why the suppressor strains restore auxin-related pathways. However, it remains unclear whether the observed restored auxin signaling was the result of the activation of BZR1/BES1 or whether the suppressors employed other pathways to restore the auxin signaling. On the other hand, at low concentrations of BRs, BIN2 enhances the DNA-binding activity of the auxin response factors ARF2, ARF7, and ARF19 through phosphorylation. This results in growth and root elongation in the absence of BRs [37, 41]. Hence, optimal plant growth and development regulated by auxin signaling requires a balanced level of BR signaling. Like with $\mathrm{ABA}$ and auxin, crosstalk between BRs and respectively ethylene and cytokinin has also been reported [28].

BR signaling controls development and growth by regulating metabolic processes such as fatty acid metabolism. For example, exogenous BR treatment was shown to promote leaf senescence, likely via the alteration of fatty acid composition in Pisum sativum [42]. Exogenous BR treatment would increase the content of free fatty acids and decrease the content of fatty acids bound to galactolipids [42]. Similar studies showed the effect of exogenous BR on fatty acid composition under salt [43] and drought [44] stress. As expected, fatty acid metabolism (subnetwork 3) and developmental processes (subnetwork 4) have been partially restored by bri1-5 suppressor lines, supporting the impact of BR signaling on the development, and the composition of fatty acids.

Transcription factors, regulators, oligopeptides, and proteins are an essential part of BR- and other signaling pathways. Previous studies showed that BR treatment increases the protein levels in the nuclei of hypocotyl cells $[11,45]$. Transport of transcription factors and regulators to the nucleus is essential to regulate gene expression by BR signaling as a downstream effect [46]. This is in line with our results: oligopeptide transport (subnetwork 5) is affected in the bri1-5 mutant and is partially restored by suppressor lines.

\section{Negative feedback between BR signaling and BR biosynthesis}

In addition, our results provide evidence for negative feedback between BR signaling and BR biosynthesis.
This hypothesis was already made by Noguchi et al. [2] who explained the observed accumulation of BR precursors in bri1 mutants by the presence of a negative effect of the BR signaling proteins BZR1/BES1 on the BRbiosynthesis pathway. Although it cannot be excluded that the previously reported accumulation of BR precursors in bri1-5 mutants results from the inability of the mutant lines to use available BRs, our observation in BR signaling mutants suggests that aberrant regulation of BR biosynthesis can also contribute to the accumulation of BRs precursors: it seems that non-aberrant BR signaling is required for homeostasis of appropriate levels of endogenous BRs. Our results also show that the level of negative feedback depends on the degree to which the suppressor could compensate for the phenotypic difference between the bri1-5 and WS2. The better the defects in BR signaling were alleviated (as reflected by the phenotype), the less pronounced the observed effects on the BR biosynthetic genes. This observation also supports the hypothesis made by Gruszka et al. [4] that BIN2 would regulate BR-biosynthesis through phosphorylating CESTA, a transcription factor that regulates BRbiosynthesis (Fig. 1).

\section{BR signaling and stress response}

Because BR signaling regulates response to a wide spectrum of stresses [15], it is not unexpected we observed that stress response genes were affected in the mutants that interfered with BR signaling. At the low level of BRs (or BR signaling deficiency e.g. bri1-5) the activated SnRK2 by BIN2 mimics the presence of ABA, activates $\mathrm{ABI} 5$, and finally regulates stress response genes (Fig. 1). In the presence of BRs, BZR1/BES1 inhibits ABI5 and can terminate the ABA signaling. On the other hand, BZR1/BES1 can regulate the expression of stress response independent of ABA (Fig. 1). Therefore, some stress response genes are regulated by BIN2 at low levels of BRs through ABA signaling, while other stresses are controlled by BZR1/BES1 at high levels of BRs independent of ABA signaling. We observed that by partially recovering bri1-5 signaling deficiency in bri1-5 suppressor strains, the transcript level of some stressresponse genes is restored to normal, but other stressresponse genes become induced. The need for optimal BR homeostasis might also explain why some gain-offunction mutants (e.g. bes1-D) described in the literature or why treatment with exogenous BR give rises to a phenotypic response that is worse than the one observed in the bri1-5 mutant (shorter root) [47]. This further confirms that an appropriate balance in BR signaling is essential to guarantee coherent cross-talk between hormones signaling networks and any aberration of this optimal level leads to activation of stress response genes. 
In addition, there is evidence that BAK1 plays a role in regulating stress-response pathways independently from BR signaling [16]. Since BAK1 usually works as a coreceptor and serves to promote cross-phosphorylation leading to downstream signaling, the existence of other stress-sensor receptors interacting with BAK1 cannot be excluded.

\section{Acidification possibly involved in providing an optimal environment for BRI1 and ligand binding}

Our analysis of the genes/pathways that are uniquely involved in the bri1-5/brs $1-1 D$ suppressor line to compensate for the bri1-5 mutant showed that BRS1 seems involved in the acidification of the apoplast environment. We hypothesize that this acidification could contribute to an improved BRI1-BRs binding or BRI1-BAK1 dimerization and hence restoration of the bri1-5 mutant phenotype. In vitro studies have indeed shown that BRs preferentially bind to BRI1 in an acidic cell wall environment $(\mathrm{pH}<5)[26,48]$. It has also been suggested that changing the $\mathrm{pH}$ environment by endocytosis of BRI1 from the plasma membrane into the cytosol reduces the affinity of BRI1 to BRs and would terminate BR signaling [26]. The same ligand-receptor mechanism has been reported in animal cells [49]. In addition, acidification of the apoplast is the major requirement for increasing cell wall extensibility, which controls cell extension and can also be a compensatory pathway in brs1-1D [50]. This is confirmed by the phenotypic analysis which shows that indeed the bri1-5/brs $1-1 D$ line at least partially restores the epidermal cell length (Fig. 2). These observations support that the brs $1-1 D$ mutant can restore BR signaling by creating an acidic environment and providing the optimal conditions for either BRI1-ligand binding or BRI1-BAK1 dimerization along with improving cell wall extensibility. This might also explain the very similar genome-wide expression impact of brs1-1D and bri1$1 D$ and explains why overexpression of BRS1 can suppress two weak BRI1 extracellular domain mutants, bri1-5 and bri1-9, but not the strong cytoplasmic domain mutant bri1-1.

The bri1-5/brs1-1D suppressor line also induces glutathione transferase activity which is necessary for redox homeostasis. This link between BR and redox signaling is in line with the literature [51]. An oxidative environment induces BZR1 activity and promotes the interaction of BZR1 with downstream TFs, ARF6, and PIF4 [51]. Since a loss-of-function mutant of BRS1 shows no obvious phenotype, [3] but its gain-of-function mutant does and partially restores bri1-5 signal deficiency, it can be suggested that BRS1 does not have a regulatory role and only provides a better condition for triggering the BR signaling by making the apoplast environment more acidic.

\section{Conclusions}

In this study, we performed expression, pathway, and network analysis to provide more insight into the BR signaling by taking advantage of the availability of mutants for keys genes in BR signaling. Our results suggest that ABA signaling plays a significant role in alleviating the bri1-5 dwarf phenotype. The fact that also other phytohormone signaling pathways are restored to the wild-type expression level in all bri1-5 suppressors confirms the crosstalk between BR and other phytohormone signalings. The negative feedback from BR signaling on BR biosynthesis was also confirmed by quantitative evidence. In addition, a new indirect role for BRS1 in BR signaling was suggested. However, our study is limited to transcriptome analysis and BR signaling is likely regulated to a large extent at the post-transcriptional level (i.e. phosphorylation). By using network analysis we can partially deal with this missing information, but this approach is limited by the incompleteness of the interaction network. Hence, more extensive validation studies are required to confirm our hypotheses. Besides, this study mainly focuses on the suppressor lines of bri1-5. Future work by adding enhancer lines such as bri1-5/ bri1-1, bri1-5/brs1-1, and bri1-5/bak1-1 or by studying the effect of suppressors not only in the BRI1 as a loss-of-function background but also using double or triple mutants of multiple genes of the BRI1 gene family and/or BRI1-like genes [52] could be interesting to further elucidate the mechanism of $B R I$ signaling. In addition, future research by scRNA-seq of bri1-5 suppressor mutants across different time points of hypocotyl growth would be key to elucidating the detailed mechanisms of BR signaling at the level of cell types [53, 54].

\section{Methods}

\section{Expression profiling experiment and differential expression analysis}

The two activation tagging suppressors of bri1-5, bri1$5 / b a k 1-1 D$, and bri1-5/brs1-1D were obtained from our previous study [12]. An additional activation tagging suppressor line bri1-5/bri1-1D was generated in this study as previously described [3]. Wild-type (WS2), the loss-of-function BR mutant (bri1-5), and its three suppressor mutants (bri1-5/brs1-1D, bri1-5/bak1-1D, bri1-5/bri1-1D) were grown at $22^{\circ} \mathrm{C}$ in a long-day condition ( $16 \mathrm{~h}$ of light and $8 \mathrm{~h}$ of dark) in a greenhouse for 7 days. All mutants were generated from the WS2 ecotype background. Microarray analysis of all genotypes was performed with three biological replicates. Total RNA of 7-day whole seedlings was isolated, labeled, and hybridized with an Arabidopsis ATH1 genome array according to the Affymetrix instructions. Scanning of the array was performed using the Agilent GeneArray 
Scanner. The data are available in GEO (GSE70843). The CEL files were preprocessed using the AFFY package (background correction, quantile normalization, and probe value summarization (RMA normalization)) [55]. The arrayQualityMetrics package was used to check the quality of the normalized expression values [56]. All samples passed the quality check. Non-unique probe sets were removed, and the expression value of genes was calculated as the average of the expression measured by the probe sets that covered the gene. The consistency between replicate samples was assessed using PCA. Differential expression was calculated by comparing the samples of the mutated lines with those obtained from the wild-type (WS2) using the Limma package [57]. To define differentially expressed genes, the absolute fold change and false discovery rate (FDR) threshold were set at 1.5 and 0.05 , respectively resulting in a total of 1413 differentially expressed genes. Further, eight differentially expressed genes were randomly selected and confirmed by RT-qPCR following standard protocol with three biological and three technical repeats [58]. The primers used for qPCR are presented in Table S3. Pathway enrichment analysis was carried out using the MapMan software [59] and GO enrichment was performed using TAIR GO enrichment [60].

\section{Phenotypic analysis}

The phenotypic impact of suppressor lines was evaluated through the measurement of the root, hypocotyl, and epidermal cell length on the 7-day old seedlings. The root and hypocotyl lengths were measured on 40 plants and hypocotyl epidermal cells were scanned using the electron microscope (SEM) on samples taken from 3 random plants for each line. The mean of epidermal cell length per image was determined using Fiji software [61]. Statistical analysis was performed using One-way ANOVA followed by Tukey's multiple comparisons in R [62].

\section{Retrieving BR-responsive genes}

We performed a literature study to compile a list of "high-confidence" BR-responsive genes, i.e. genes regulated by exogenous BR treatment or in a line containing a gain-of-function mutation in a BR signaling gene with consistent transcriptional response from at least five references [31, 32, 63-71]. A full list of marker genes together with the references in which they were found and an indication of their expression behavior upon addition of external BRs (or gain of mutation in BR signaling genes) is given in supplementary file (Additional file 2). The intersection of the genes that were differentially expressed in the bri1-5 mutant line with the list of "high-confidence" BR-responsive genes was used to evaluate the consistency between the expression data of our study and literature and to determine which suppressor best restored the expression of those genes to WS2 level.

\section{Network analysis}

A high confidence Arabidopsis interaction network was compiled from the following sources: 64185 FunTFBS regulatory interactions were obtained from PlantRegMap [57], 96,827 protein-protein interactions from AtPIN [72] and 34,003 metabolic interactions from KEGG [73]. This resulted in a final number of 182,748 interactions between 21,263 unique genes. In this integrated network, nodes represented genes and edges the interactions between the genes.

To perform network analysis with PheNetic [24] network edges need to be weighted. The weight is derived from the $\log 2$ fold change $(\operatorname{logFC})$ expression of a gene as indicated below. To each gene, we assigned as logFC, the highest value that was observed for this gene across the assessed mutant lines as compared to WS2. To assign a $p$-value to this $\operatorname{logFC}$, we empirically estimated the distribution of the observed max $\log F C$ for all genes. As we expected that most of the genes would not change their expression this is an estimate of the null distribution. The mean $(\mu)$ and standard deviation $(\sigma)$ of this distribution was estimated empirically using maximum likelihood implemented in the MASS package [74]. Using the values of the mean and the standard deviation each gene was assigned a significance score (gene-score) based on a two-tailed T-test which reflects the degree to which the gene has been affected as compared to other genes as follow [24]:

gene _score $=\operatorname{abs}\left(1-2 * \phi_{(\mu, \sigma)} \max (\log F C)\right)$.

The edge weight between a source $(\mathrm{S})$ and target $(\mathrm{T})$ nodes was derived by the product of the $\mathrm{S}$ gene-score and $\mathrm{T}$ gene-score.

This weighted interaction network was used together with the seed gene list (list of differentially expressed genes) in PheNetic [23, 24]. PheNetic aims at connecting as many genes as possible from the seed list on the interaction network in the most parsimonious way (using the least number of edges). By enforcing such a parsimonious solution, PheNetic detects subnetworks in which genes from the seed list are closely connected. Such connected components can be viewed as proxies of pathways. The PheNetic expression subcommand was run in the downstream mode with the following parameters: min cost: 0.1 ; max cost: 5 ; step size: log scale between $\max$ and min cost with 28 steps; path-length $=4$; k-best paths: 50; for all other parameters the default values were used. For each edge cost, the highest-scoring subnetwork was selected. Furthermore, the Jaccard index of all the subnetworks with the same edge cost was computed. For each cost, the subnetwork is rejected if it has a low stability score (i.e. Jaccard index smaller than 0.5) 
or if it is too large (more than 500 interactions). The final subnetworks are then the union of all the "best subnetworks" for each edge penalty that passed the stability and size requirements.

\section{Gene groups used for network analysis}

The gene groups used for network analysis were derived from the Venn diagram displayed in Fig. 3. To perform network analysis, we defined respectively restoring genes (group A, genes with altered expression in the bri1-5 mutant, but restored to WS2 level in at least 2 suppressors), compensatory genes (group B, (genes not differentially expressed in the bri1-5 mutant, but differentially expressed in at least two suppressor strains), nonrestored genes (group $\mathrm{C}$, genes that differentially expressed in the bri1-5 mutant and at least two of the suppressor strains) and genes that are uniquely affected in respectively the bri1-5/brs1-1D (group D), the bri15/bak1-1D (group F), and the bri1-5/bri-1D (group E) (Additional file 1).

To study the interaction between restored, compensatory, and non-restored genes, we combined the genes of groups A, B, and C (789 genes) to perform network analysis. Each group consists of its core genes. The core of group A (restored genes) consists of 118 genes that are restored to the wild-type state by all suppressors. The core of group B (compensatory genes) consists of 23 genes, i.e. genes that were significantly differentially expressed in all three bri1-5 suppressors, but not in bri1-5 mutant. The core of group C (non-restored genes) consists of 149 genes that are affected in bri1-5 mutant but not restored by any suppressors. However, as the size of the gene sets is dependent on the choice of an arbitrary threshold, we assumed that some of the genes belonging to the processes represented by these core gene sets were found to be significantly differentially expressed in two of the three lines only (and slightly below the threshold in the third). That is why we extended the core gene sets with the genes that were differentially expressed in at least two lines, rather than in all three of them. Extended gene sets A, B and $\mathrm{C}$ were subsequently combined to study the interaction between the restored, compensatory, and non-restored genes.

\footnotetext{
Abbreviations

BR: Brassinosteroid; BRI: BR insensitive; BRL1: BRI1-like homologs; BAK1: BRI1Associated receptor kinase; ABA: Abscisic acid; BIN: Brassinosteroid insensitive; BRZ: Brassinazole-Resistant; BES: Br-Insensitive-Ems-Suppressor; PP: Phosphatase; BRS: BRI suppressor; SCP: Serine carboxypeptidase; ECS: Extra Carpels and seeds; FLS: Flagellin-Sensitive; RT-qPCR: Real time quantitative polymerase chain reaction; PCA: Principal component analysis; ROS: Reactive oxygen species; FDR: False discovery rate; TF: Transcription Factor; FunTFBS: Functional transcription binding site; AtPIN: Arabidopsis thaliana protein interaction network
}

\section{Supplementary Information}

The online version contains supplementary material available at https://doi. org/10.1186/s12864-021-07778-w.

Additional file 1. List of differentially expressed genes as compared to WS2 in each line and group membership. (XLSM 104 kb)

Additional file 2. List of marker genes being differentially expressed upon addition of external BRs or in line with a gain of mutation in BR signaling genes at least in 5 studies.

Additional file 3. $\mathrm{GO}$ enrichment for genes exclusively differentially expressed in each suppressor.

Additional file 4: Fig. S1. The T-DNA insertion site for bri1-1D (A), and microscopic images of 7-day old hypocotyl cells for WS2 (B), bri1-5 (C), bri1-5/bak1-1D (D), bri1-5/bri1-1D (E), bri1-5/brs1-1D (F). Fig. S2. PCA plot for assessing the reproducibility of the gene expression dataset. Samples taken from the same genotype are represented in the same color. The plot indicates high consistency between replicate samples as they are located close to each other when plotted on the first and second principal components. Fig. S3. RT-qPCR results for relative expression of selected genes and their corresponding values from microarray analysis. The values represent the log2 of relative expression (sample1/sample2). Rows indicate gene names and columns show the comparison between the indicated lines. Columns with pink header represent the RT-qPCR values, and columns with yellow header are microarray measurements. The red color on the heatmap indicates that the gene has been upregulated in sample1 as compared to sample2, while blue indicates down-regulation. Fig. S4. Comparing genome-wide expression impact between bri1-5 suppressor lines. Fig. S5. Heatmap of expression of the marker genes that up/down regulation of their expression was confirmed by at least 5 independent references and also affected in the bri1-5 line of our study. For each line, the row-scaled normalized expression data of the 3 biological replicates are shown as adjacent columns. In each row the gradient red color indicates the higher expression for the gene compared to other samples while blue indicates the lower expression. Fig. S6. Pathway analysis (MapMan metabolism) showing for each mutant line the expression changes compared to WS2. Panel A: bri1-5, Panel B: bri1-5/bri1-1D, Panel C: bri1-5/brs1-1D, Panel D: bri1-5/bak1-1D. Fig. S7. Pathway analysis (MapMan: large enzyme families) showing for each mutant line the expression changes compared to WS2. Panel A: bri1-5, Panel B: bri1-5/bri1-1D, Panel C: bri1-5/brs1-1D, Panel D: bri1-5/bak1-1D. Fig. S8. Pathway analysis (MapMan: gene regulation) showing for each mutant line the expression changes compared to WS2. Panel A: bri1-5, Panel B: bri1-5/bri1-1D, Panel C: bri1-5/brs1-1D, Panel D: bri1-5/bak1-1D. Fig. S9. Expression pattern in each mutant line of genes related to ABA signaling, Glutathione metabolism, and ion related hemostasis as discussed in the main text. Mutant lines are represented in the $x$-axis. The $y$-axis indicates the $\log 2$ normalized expression value of the gene. Table S1. RTqPCR test of log-fold change (log-FC) of the genes that are overexpressed by activation-tagging in the suppressors at the 7 days seedling stage.

Table S2. Summary of the most significant results obtained by MapMan pathway analysis (metabolism, regulation and, large-enzyme families overview). Left column: enriched pathways; entries provide for each line the degree to which the pathway is enriched. P-values are FDR corrected using Benjamini-Hochberg). Table S3. Designed primers for RT-qPCR.

\section{Acknowledgments}

We would like to thank the anonymous reviewers for the critical remarks which largely improved our manuscript.

\section{Authors' contributions}

Conceptualization: J. L, T.S., R.S.R., K.M.; Methodology: T.S., R.S.R.; Investigation: D.Z., X.G., J.Y., G.M; Writing - Original Draft: T.S., R.S.R, K.M; Writing - Review \& Editing: J.L., K.M., G.M; Funding Acquisition: J.L., K. M, R.S.R., T.S.; Resources: J.L.; Supervision: K.M., J.L. All authors read and approved the final manuscript.

\section{Funding}

This work was supported by the National Basic Research Program of China [grant 31720103902 and 31530005 to J.L., grant 31700197 to T.S.], Youth Innovation Promotion Association of the Chinese Academy of Sciences 
[grant 2019335 to T.S.] and the Ministry of Science, Research and Technology, Iran [grant to R.SR]. The work was also supported by grants of the Fonds Wetenschappelijk Onderzoek-Vlaanderen (FWO) [G046318, G.0371.06, and 3G045620] and UGent Bijzonder onderzoeksfonds.

\section{Availability of data and materials}

The microarray datasets have been deposited in GEO under accession number GSE70843. Requests for resources, codes, and material should be directed to and will be fulfilled by the Lead Contact, Jia Li (lijia@lzu.edu.cn).

\section{Declarations}

Ethics approval and consent to participate Not applicable.

\section{Consent for publication}

Not applicable.

\section{Competing interests}

The authors declare that they have no competing interests.

\section{Author details}

'Department of Plant Biotechnology and Bioinformatics, Ghent University, Ghent, Belgium. ${ }^{2}$ Department of Information Technology, IDLab, imec, Ghent University, Ghent, Belgium. ${ }^{3}$ Key Laboratory of Aquatic Botany and Watershed Ecology, Wuhan Botanical Garden, Chinese Academy of Sciences, Wuhan 430074, China. ${ }^{4}$ Ministry of Education Key Laboratory of Cell Activities and Stress Adaptations, School of Life Sciences, Lanzhou University, Lanzhou 730000, China. ${ }^{5}$ Department of Biochemistry, Genetics and Microbiology, University of Pretoria, Pretoria, South Africa.

\section{Received: 9 September 2020 Accepted: 7 June 2021}

Published online: 22 June 2021

\section{References}

1. Clouse SD, Sasse JM. BRASSINOSTEROIDS: essential regulators of plant growth and development. Annu Rev Plant Physiol Plant Mol Biol. 1998;49(1): 427-51. https://doi.org/10.1146/annurev.arplant.49.1.427.

2. Noguchi T, Fujioka S, Choe S, Takatsuto S, Yoshida S, Yuan H, et al. Brassinosteroid-insensitive dwarf mutants of Arabidopsis accumulate brassinosteroids. Plant Physiol. 1999;121(3):743-52. https://doi.org/10.1104/ pp.121.3.743.

3. Li J, Lease KA, Tax FE, Walker JC. BRS1, a serine carboxypeptidase, regulates BRI1 signaling in Arabidopsis thaliana. Proc Natl Acad Sci U S A. 2001;98(10): 5916-21. https://doi.org/10.1073/pnas.091065998.

4. Gruszka D. The brassinosteroid signaling pathway-new key players and interconnections with other signaling networks crucial for plant development and stress tolerance. Int J Mol Sci. 2013;14(5):8740-74. https:// doi.org/10.3390/ijms14058740.

5. Planas-Riverola A, Gupta A, Betegon-Putze I, Bosch N, Ibanes M, CanoDelgado Al. Brassinosteroid signaling in plant development and adaptation to stress. Development. 2019;146(5).

6. Li J, Chory J. A putative leucine-rich repeat receptor kinase involved in brassinosteroid signal transduction. Cell. 1997;90(5):929-38. https://doi.org/1 0.1016/S0092-8674(00)80357-8.

7. Kinoshita T, Cano-Delgado A, Seto H, Hiranuma S, Fujioka S, Yoshida S, et al. Binding of brassinosteroids to the extracellular domain of plant receptor kinase BRI1. Nature. 2005;433(7022):167-71. https://doi.org/10.1038/na ture03227.

8. Nam KH, Li J. BRI1/BAK1, a receptor kinase pair mediating brassinosteroid signaling. Cell. 2002;110(2):203-12. https://doi.org/10.1016/S0092-8674(02 )00814-0.

9. Wang X, Kota U, He K, Blackburn K, Li J, Goshe MB, et al. Sequential transphosphorylation of the BRI1/BAK1 receptor kinase complex impacts early events in brassinosteroid signaling. Dev Cell. 2008;15(2):220-35. https:// doi.org/10.1016/j.devcel.2008.06.011.

10. Chen W, LV M, Wang Y, Wang PA, Cui Y, Li M, et al. BES1 is activated by EMS1-TPD1-SERK1/2-mediated signaling to control tapetum development in Arabidopsis thaliana. Nat Commun. 2019;10(1):4164. https://doi.org/10.1038/ s41467-019-12118-4.
11. Wang Z-Y, Nakano T, Gendron J, He J, Chen M, Vafeados D, et al. Nuclearlocalized BZR1 mediates brassinosteroid-induced growth and feedback suppression of brassinosteroid biosynthesis. Dev Cell. 2002;2(4):505-13. https://doi.org/10.1016/\$1534-5807(02)00153-3.

12. Li J, Wen J, Lease KA, Doke JT, Tax FE, Walker JC. BAK1, an Arabidopsis LRR receptor-like protein kinase, interacts with BRI1 and modulates brassinosteroid signaling. Cell. 2002;110(2):213-22. https://doi.org/10.1016/ S0092-8674(02)00812-7.

13. Wen J, Li J, Walker JC. Overexpression of a serine carboxypeptidase increases carpel number and seed production in a rabidopsis thaliana. Food and Energy Security. 2012;1(1):61-9. https://doi.org/10.1002/fes3.5.

14. Nolan TM, Brennan B, Yang M, Chen J, Zhang M, Li Z, et al. Selective Autophagy of BES1 Mediated by DSK2 Balances Plant Growth and Survival. Dev Cell. 2017:41(1):33-46.e7.

15. Peres A, Soares JS, Tavares RG, Righetto G, Zullo MAT, Mandava NB, et al. Brassinosteroids, the Sixth Class of Phytohormones: A Molecular View from the Discovery to Hormonal Interactions in Plant Development and Stress Adaptation. Int J Mol Sci. 2019;20(2):331.

16. Chinchilla D, Zipfel C, Robatzek S, Kemmerling B, Nurnberger T, Jones JD, et al. A flagellin-induced complex of the receptor FLS2 and BAK1 initiates plant defence. Nature. 2007:448(7152):497-500. https://doi.org/10.1038/na ture05999.

17. Kemmerling B, Schwedt A, Rodriguez P, Mazzotta S, Frank M, Qamar SA, et al. The BRI1-associated kinase 1, BAK1, has a brassinolide-independent role in plant cell-death control. Curr Biol. 2007;17(13):1116-22. https://doi. org/10.1016/j.cub.2007.05.046.

18. Li J. Multi-tasking of somatic embryogenesis receptor-like protein kinases. Curr Opin Plant Biol. 2010;13(5):509-14. https://doi.org/10.1016/j.pbi.2010.09.004

19. Albrecht C, Boutrot F, Segonzac C, Schwessinger B, Gimenez-lbanez S, Chinchilla $\mathrm{D}$, et al. Brassinosteroids inhibit pathogen-associated molecular pattern-triggered immune signaling independent of the receptor kinase BAK1. Proc Natl Acad Sci U S A. 2012;109(1):303-8. https://doi.org/10.1073/ pnas.1109921108.

20. Kim BH, Kim SY, Nam KH. Genes encoding plant-specific class III peroxidases are responsible for increased cold tolerance of the brassinosteroidinsensitive 1 mutant. Mol Cell. 2012:34(6):539-48. https://doi.org/10.1007/s1 0059-012-0230-z.

21. Kim SY, Kim BH, Lim CJ, Lim CO, Nam KH. Constitutive activation of stressinducible genes in a brassinosteroid-insensitive 1 (bri1) mutant results in higher tolerance to cold. Physiol Plant. 2010;138(2):191-204. https://doi. org/10.1111/j.1399-3054.2009.01304.x.

22. Guo H, Li L, Aluru M, Aluru S, Yin Y. Mechanisms and networks for brassinosteroid regulated gene expression. Curr Opin Plant Biol. 2013;16(5): 545-53. https://doi.org/10.1016/j.pbi.2013.08.002

23. De Maeyer D, Renkens J, Cloots L, De Raedt L, Marchal K. PheNetic: network-based interpretation of unstructured gene lists in E. coli. Mol BioSyst. 2013:9(7):1594-603. https://doi.org/10.1039/c3mb25551d.

24. De Maeyer D, Weytjens B, Renkens J, De Raedt L, Marchal K. PheNetic: network-based interpretation of molecular profiling data. Nucleic Acids Res. 2015;43(W1):W244-50. https://doi.org/10.1093/nar/gkv347.

25. Cai Z, Liu J, Wang H, Yang C, Chen Y, Li Y, et al. GSK3-like kinases positively modulate abscisic acid signaling through phosphorylating subgroup III SnRK2s in Arabidopsis. Proc Natl Acad Sci. 2014;111(26):9651-6. https://doi. org/10.1073/pnas.1316717111.

26. Belkhadir $Y$, Jaillais $Y$. The molecular circuitry of brassinosteroid signaling. New Phytol. 2015;206(2):522-40. https://doi.org/10.1111/nph.13269.

27. Takahashi Y, Zhang J, Hsu P-K, Ceciliato PH, Zhang L, Dubeaux G, et al. MAP 3Kinase-dependent SnRK2-kinase activation is required for abscisic acid signal transduction and rapid osmotic stress response. Nat Commun 2020; 11(1):1-12, 12, DOl: https://doi.org/10.1038/s41467-019-13875-y.

28. Choudhary SP, Yu J-Q, Yamaguchi-Shinozaki K, Shinozaki K, Tran L-SP. Benefits of brassinosteroid crosstalk. Trends Plant Sci. 2012;17(10):594-605. https://doi.org/10.1016/j.tplants.2012.05.012.

29. Tian $H$, Lv B, Ding T, Bai M, Ding Z. Auxin-BR interaction regulates plant growth and development. Front Plant Sci. 2018:8:2256. https://doi.org/10.33 89/fpls.2017.02256.

30. Fujioka S, Yokota T. Biosynthesis and metabolism of brassinosteroids. Annu Rev Plant Biol. 2003;54(1):137-64. https://doi.org/10.1146/annurev.arplant.54. 031902.134921.

31. Goda H, Sawa S, Asami T, Fujioka S, Shimada Y, Yoshida S. Comprehensive comparison of auxin-regulated and brassinosteroid-regulated genes in 
Arabidopsis. Plant Physiol. 2004;134(4):1555-73. https://doi.org/10.1104/pp.1 03.034736 .

32. Yu X, Li L, Zola J, Aluru M, Ye H, Foudree A, et al. A brassinosteroid transcriptional network revealed by genome-wide identification of BESI target genes in Arabidopsis thaliana. Plant J. 2011;65(4):634-46. https://doi. org/10.1111/j.1365-313X.2010.04449.X.

33. Kumar K, Askari F, Sahu MS, Kaur R. Candida glabrata: a lot more than meets the eye. Microorganisms. 2019;7(2):39. https://doi.org/10.3390/microorga nisms7020039.

34. Michelet B, Boutry M. The plasma membrane H+-ATPase (a highly regulated enzyme with multiple physiological functions). Plant Physiol. 1995;108(1):16. https://doi.org/10.1104/pp.108.1.1.

35. Wang H, Tang J, Liu J, Hu J, Liu J, Chen Y, et al. Abscisic acid signaling inhibits brassinosteroid signaling through dampening the dephosphorylation of BIN2 by ABI1 and ABI2. Mol Plant. 2018;11(2):315-25. https://doi.org/10.1016/j.molp.2017.12.013.

36. Kulik A, Wawer I, Krzywińska E, Bucholc M, Dobrowolska G. SnRK2 protein kinases - key regulators of plant response to abiotic stresses. Omics: a journal of integrative biology. 2011;15(12):859-72. https://doi.org/10.1089/ omi.2011.0091.

37. Lin Z, Li Y, Zhang Z, Liu X, Hsu C-C, Du Y, et al. A RAF-SnRK2 kinase cascade mediates early osmotic stress signaling in higher plants. Nat Commun. 2020;11(1):1-10.

38. K-i M, Miyakawa T, Sawano Y, Kubota K, Kang H-J, Asano A, et al. Structural basis of abscisic acid signalling. Nature. 2009;462(7273):609-14.

39. Zhou X-Y, Song L, Xue H-W. Brassinosteroids regulate the differential growth of Arabidopsis hypocotyls through auxin signaling components IAA19 and ARF7. Mol Plant. 2013;6(3):887-904. https//doi.org/10.1093/mp/sss 123.

40. Liu K, Li Y, Chen X, Li L, Liu K, Zhao H, et al. ERF72 interacts with ARF6 and BZR1 to regulate hypocotyl elongation in Arabidopsis. J Exp Bot. 2018. 69(16):3933-47. https://doi.org/10.1093/jxb/ery220.

41. Cho H, Ryu H, Rho S, Hill K, Smith S, Audenaert D, et al. A secreted peptide acts on BIN2-mediated phosphorylation of ARFs to potentiate auxin response during lateral root development. Nat Cell Biol. 2014;16(1):66-76. https://doi.org/10.1038/ncb2893.

42. Fedina E, Yarin A, Mukhitova F, Blufard A, Chechetkin I. Brassinosteroid-induced changes of lipid composition in leaves of Pisum sativum $L$ during senescence. Steroids. 2017;117:25-8. https://doi.org/10.1016/.jsteroids.2016.10.009.

43. Pokotylo I, Kretynin S, Khripach V, Ruelland E, Blume YB, Kravets V. Influence of 24epibrassinolide on lipid signalling and metabolism in Brassica napus. Plant Growth Regul. 2014;73(1):9-17. https//doi.org/10.1007/s10725-013-9863-y.

44. Zafari M, Ebadi A, Sedghi M, Jahanbakhsh S. Alleviating effect of 24epibrassinolide on seed oil content and fatty acid composition under drought stress in safflower. J Food Compos Anal. 2020;92:103544. https:// doi.org/10.1016/j.jfca.2020.103544

45. Yin Y, Wang Z-Y, Mora-Garcia S, Li J, Yoshida S, Asami T, et al. BES1 accumulates in the nucleus in response to brassinosteroids to regulate gene expression and promote stem elongation. Cell. 2002;109(2):181-91. https://doi.org/10.1016/S0092-8674(02)00721-3.

46. Ryu H, Kim K, Cho H, Hwang I. Predominant actions of cytosolic BSU1 and nuclear BIN2 regulate subcellular localization of BES1 in brassinosteroid signaling. Mol Cell. 2010;29(3):291-6. https:/doi.org/10.1007/s10059-010-0034-y.

47. González-García M-P, Vilarrasa-Blasi J, Zhiponova M, Divol F, Mora-García S, Russinova $\mathrm{E}$, et al. Brassinosteroids control meristem size by promoting cell cycle progression in Arabidopsis roots. Development. 2011;138(5):849-59. https://doi.org/10.1242/dev.057331.

48. She J, Han Z, Kim T-W, Wang J, Cheng W, Chang J, et al. Structural insight into brassinosteroid perception by BRI1. Nature. 2011;474(7352):472-6. https://doi.org/10.1038/nature10178.

49. Maxfield FR, McGraw TE. Endocytic recycling. Nat Rev Mol Cell Biol. 2004; 5(2):121-32. https://doi.org/10.1038/nrm1315.

50. Hager A. Role of the plasma membrane H+-ATPase in auxin-induced elongation growth: historical and new aspects. J Plant Res. 2003;116(6):483505. https://doi.org/10.1007/s10265-003-0110-x.

51. Lv B, Tian H, Zhang F, Liu J, Lu S, Bai M, et al. Brassinosteroids regulate root growth by controlling reactive oxygen species homeostasis and dual effect on ethylene synthesis in Arabidopsis. PLoS Genet. 2018;14(1):e1007144. https://doi.org/10.1371/journal.pgen.1007144.

52. Lozano-Elena F, Caño-Delgado Al. Emerging roles of vascular brassinosteroid receptors of the BRl1-like family. Curr Opin Plant Biol. 2019, 51:105-13. https://doi.org/10.1016/j.pbi.2019.06.006.
53. Liu Q, Liang Z, Feng D, Jiang S, Wang Y, Du Z, et al. Transcriptional Landscape of Rice Roots at the Single Cell Resolution. Molecular Plant. 2021; 14(3);384-94.

54. Zhang T-Q, Xu Z-G, Shang G-D, Wang J-W. A single-cell RNA sequencing profiles the developmental landscape of Arabidopsis root. Mol Plant. 2019; 12(5):648-60. https://doi.org/10.1016/j.molp.2019.04.004.

55. Gautier L, Cope L, Bolstad BM, Irizarry RA. Affy-analysis of Affymetrix GeneChip data at the probe level. Bioinformatics. 2004;20(3):307-15. https:// doi.org/10.1093/bioinformatics/btg405.

56. Kauffmann A, Gentleman R, Huber W. arrayQualityMetrics--a bioconductor package for quality assessment of microarray data. Bioinformatics. 2009; 25(3):415-6. https://doi.org/10.1093/bioinformatics/btn647.

57. Ritchie ME, Phipson B, Wu D, Hu Y, Law CW, Shi W, et al. limma powers differential expression analyses for RNA-sequencing and microarray studies. Nucleic Acids Res. 2015;43(7):e47.

58. Wong ML, Medrano JF. Real-time PCR for mRNA quantitation. Biotechniques. 2005;39(1):75-85. https://doi.org/10.2144/05391RV01.

59. Thimm O, Bläsing O, Gibon Y, Nagel A, Meyer S, Krüger P, et al. MAPMAN: a user-driven tool to display genomics data sets onto diagrams of metabolic pathways and other biological processes. Plant J. 2004;37(6):914-39. https:/ doi.org/10.1111/j.1365-313X.2004.02016.X.

60. Lamesch P, Berardini TZ, Li D, Swarbreck D, Wilks C, Sasidharan R, et al. The Arabidopsis information resource (TAIR): improved gene annotation and new tools. Nucleic Acids Res. 2012;40(D1):D1202-D10. https://doi.org/10.1 093/nar/gkr1090.

61. Schindelin J, Arganda-Carreras I, Frise E, Kaynig V, Longair M, Pietzsch T, et al. Fiji: an open-source platform for biological-image analysis. Nat Methods. 2012;9(7):676-82. https://doi.org/10.1038/nmeth.2019.

62. Team RC. R: a language and environment for statistical computing. Vienna: Austria; 2013

63. Goda H, Shimada Y, Asami T, Fujioka S, Yoshida S. Microarray analysis of brassinosteroid-regulated genes in Arabidopsis. Plant Physiol. 2002;130(3): 1319-34. https://doi.org/10.1104/pp.011254.

64. Müssig C, Fischer S, Altmann T. Brassinosteroid-regulated gene expression Plant Physiol. 2002;129(3):1241-51. https://doi.org/10.1104/pp.011003.

65. Nemhauser JL, Mockler TC, Chory J. Interdependency of brassinosteroid and auxin signaling in Arabidopsis. PLoS Biol. 2004;2(9):e258. https://doi.org/1 0.1371/journal.pbio.0020258.

66. Vert G, Nemhauser IL, Geldner N, Hong F, Chory J. Molecular mechanisms of steroid hormone signaling in plants. Annu Rev Cell Dev Biol. 2005;21(1): 177-201. https://doi.org/10.1146/annurev.cellbio.21.090704.151241.

67. Mouchel CF, Osmont KS, Hardtke CS. BRX mediates feedback between brassinosteroid levels and auxin signalling in root growth. Nature. 2006; 443(7110):458-61. https://doi.org/10.1038/nature05130.

68. Nemhauser JL, Hong F, Chory J. Different plant hormones regulate similar processes through largely nonoverlapping transcriptional responses. Cell. 2006;126(3):467-75. https://doi.org/10.1016/j.cell.2006.05.050.

69. Goda H, Sasaki E, Akiyama K, Maruyama-Nakashita A, Nakabayashi K, Li W, et al. The AtGenExpress hormone and chemical treatment data set: experimental design, data evaluation, model data analysis and data access. Plant J. 2008;55(3):526-42. https://doi.org/10.1111/j.1365-313X.2008.03510.X.

70. Guo H, Li L, Ye H, Yu X, Algreen A, Yin Y. Three related receptor-like kinases are required for optimal cell elongation in Arabidopsis thaliana. Proc Natl Acad Sci. 2009;106(18):7648-53. https://doi.org/10.1073/pnas.0812346106.

71. Sun Y, Fan X-Y, Cao D-M, Tang W, He K, Zhu J-Y, et al. Integration of brassinosteroid signal transduction with the transcription network for plant growth regulation in Arabidopsis. Dev Cell. 2010;19(5):765-77. https://doi. org/10.1016/j.devcel.2010.10.010.

72. Brandao MM, Dantas LL, Silva-Filho MC. AtPIN: Arabidopsis thaliana protein interaction network. BMC Bioinformatics. 2009;10(1):454. https://doi.org/1 0.1186/1471-2105-10-454.

73. Kanehisa M, Goto S. KEGG: Kyoto encyclopedia of genes and genomes. Nucleic Acids Res. 2000;28(1):27-30. https://doi.org/10.1093/nar/28.1.27.

74. Ripley B, Venables B, Bates DM, Hornik K, Gebhardt A, Firth D, et al. Package 'mass'. Cran r. 2013;538:113-20

\section{Publisher's Note}

Springer Nature remains neutral with regard to jurisdictional claims in published maps and institutional affiliations. 